\title{
Green Technology for Synthesis of Hierarchical Catalysts for Pharamaceutical Preparations
}

\author{
Kafiya M. Shareef ${ }^{a}$, Nadhmia Najmaddin ${ }^{b}$ Jabbar Gardyc \\ A Faculty of Nursing, Tishk International University- Erbil, 44002 Erbil, KRG-IRQ \\ ${ }^{B}$ Department of Geology, College of Science, University of Salahaddin-Erbil, 44002 Erbil, \\ KRG-IRQ. \\ c School of Chemical and Process Engineering, University of Leeds, LS2 9JT Leeds, UK.
}

\begin{abstract}
Chemists have attempted to minimize waste by designing new and more environmentally friendly methods for synthesizing catalysts. They produced an array of improved methodologies, including the use of locally available raw materials such as clay or modified clay instead of chemicals for catalyst preparation. The present study highlights the synthesis and characterization of hierarchical catalyst from locally available montmorillonite. The synthesised catalyst was characterized by, Powder X-ray diffraction, X-Ray Fluorescence (XRF), Forier Transform infrared (FT-IR) spectroscopy, $\mathrm{N}_{2}$ adsorption-desorption isotherms, Scanning electron microscopy (SEM), Thermo gravimetric analysis (TGA), for their crystal structure, surface analysis, morphology, thermal stability and catalytic performance. The synthesized catalyst designed to fit specific reaction for pharmaceutical preparation.
\end{abstract}

Keywords: clay, montmorillonite, surface area, Porosity, morphology 